\title{
Podoplanin and SOX2 expression in esophageal squamous cell carcinoma after neoadjuvant chemo-radiotherapy
}

\author{
SUSUMU SAIGUSA ${ }^{1}$, YASUHIKO MOHRI ${ }^{1}$, MASAKI OHI ${ }^{1,2}$, YUJI TOIYAMA ${ }^{1}$, YOSHITO ISHINO ${ }^{1}$, \\ YOSHINAGA OKUGAWA $^{1}$, KOJI TANAKA ${ }^{1}$, YASUHIRO INOUE ${ }^{1}$ and MASATO KUSUNOKI ${ }^{1,2}$
}

Departments of ${ }^{1}$ Gastrointestinal and Pediatric Surgery, and ${ }^{2}$ Innovative Surgery, Division of Reparative Medicine, Institute of Life Sciences, Mie University Graduate School of Medicine, 2-174 Edobashi, Tsu, Mie 514-8507, Japan

Received June 3, 2011; Accepted July 15, 2011

DOI: 10.3892/or.2011.1408

\begin{abstract}
Neoadjuvant chemo-radiotherapy (CRT) followed by surgery are the standard approaches for locally advanced esophageal cancer. However, the overall cure rate is very low. The aim of this preliminary study was to evaluate the expression of podoplanin and SOX2 known as stemness markers for esophageal squamous cell carcinoma (ESCC) and their association with clinical outcome. We obtained a total of 20 specimens from patients with ESCC who underwent neoadjuvant CRT (30-40 Gy; 5-fluorouracil plus cisplatin) followed by surgery. Podoplanin and SOX2 expression was evaluated using immunohistochemistry and the association of their expressions with clinicopathological variables was investigated. Podoplanin and SOX2 staining was detected not only in residual cancer cells, but also in the basal layer of adjacent normal mucosa after neoadjuvant CRT. High expression of podoplanin was correlated with lymph node metastasis, advanced postoperative stage and vascular invasion $(\mathrm{P}<0.05)$, while, high expression of SOX2 was correlated with lymphatic, vascular invasion, poor differentiated tumor and incomplete resection $(\mathrm{P}<0.05)$. High expression of podoplanin was significantly associated with poor overall survival $(\mathrm{P}<0.05)$. In conclusion, the expression levels of podoplanin and SOX2 expression may be useful prognostic markers for ESCC treated with neoadjuvant CRT.
\end{abstract}

\section{Introduction}

Esophageal squamous cell carcinoma (ESCC) has a particularly poor prognosis. Recent epidemiological studies have shown that the incidence is increasing (1). Neoadjuvant chemoradiotherapy followed by surgery can improve long-term

Correspondence to: Dr Susumu Saigusa, Department of Gastrointestinal and Pediatric Surgery, Division of Reparative Medicine, Institute of Life Sciences, Mie University Graduate School of Medicine, 2-174 Edobashi, Tsu, Mie 514-8507, Japan

E-mail: saigusa@clin.medic.mie-u.ac.jp

Key words: podoplanin, SOX2, esophageal squamous cell carcinoma, neoadjuvant chemo-radiotherapy survival and reduce local recurrence in patients with esophageal cancer (2). To treat incomplete local resection as well as local and distant recurrences, neoadjuvant chemotherapy alone or chemo-radiotherapy followed by surgery are the standard approaches for locally advanced esophageal cancer (3-5). However, the overall cure rate of esophageal cancer is $<20 \%$. Clarification of the mechanism of carcinogenesis and the characteristics of ESCC should contribute to developing new therapeutic strategies. Cancer stem cells (CSCs) are a small sub-population of cancer cells that have stem cell-like properties such as self-renewal and the ability to differentiate into multiple cell types $(6,7)$. CSCs are referred to as tumorinitiating cells due to these properties. Recent research suggests that CSCs are particularly resistant to conventional chemo- and radio-therapy (CRT) compared with non-CSCs. These lines of evidence prompted us to hypothesize that CSCs survived CRT and that their population was enriched after CRT. Podoplanin known as D2-40 is a mucin-type transmembrane glycoprotein and a specific marker for lymphatic endothelium. Podoplanin is a candidate CSC marker for human squamous cell carcinoma because the knockdown of podoplanin expression results in vulnerability to anticancer drugs, defective invasion and tumorigenic activity (8-10). It has been reported that a high level of podoplanin expression correlates with a poor prognosis in oral and esophageal cancer (11-13). Additionally, several authors reported that podoplanin promotes tumor cell migration leading to cancer invasion and metastasis (14). Podoplanin is also involved in lymphangiogenesis $(15,16)$. Tumor-associated lymphatic vessels act as a conduit by which disseminating cancer cells access regional lymph nodes and metastasize (17). SOX2 transcription factor reprograms genes that induce an embryonic stem cell-like (pluripotent) state in fibroblasts, which is essential for normal cell development and maintenance (18). SOX2 belongs to group B of the SOX family and plays critical roles in cell fate determination, differentiation and proliferation (19). Reduced SOX2 levels in mouse embryo models are associated with esophageal atresia and other esophageal abnormalities (20). This result suggests that SOX2 may be a stem cell marker of esophageal tissue. The goal of this preliminary and retrospective study was to evaluate the expression of podoplanin and SOX2 and their association with clinicopathological variables and to find the useful predictors of prognosis in ESCC with neoadjuvant CRT. 


\section{Materials and methods}

Patients and specimens. From 2001 to 2009, 61 patients with esophageal cancer were treated in our institute. Twenty of the 61 patients received neoadjuvant CRT followed by surgery, and all formalin-fixed, paraffin-embedded (FFPE) specimens after neoadjuvant CRT were available in this study. The study design was approved by an ethics review board. All patients signed informed consents for their tissues to be used in this study.

5-Fluorouracil and cisplatin-based chemo-radiotherapy. All patients received systemic 5-fluorouracil (5-FU) and cisplatin (CDDP) chemotherapy with concurrent radiotherapy. The regimen included four cycles of $5-\mathrm{FU}\left(600 \mathrm{mg} / \mathrm{m}^{2}\right.$ administered intravenously for $24 \mathrm{~h}$ ), and UFT (Tegafur and uracil; $400 \mathrm{mg} / \mathrm{kg}$ of body weight administered orally for five days), plus CDDP ( $4 \mathrm{mg} /$ day administered intravenously for 5 days) with concurrent 40 Gy radiation followed by surgery. Preoperative radiotherapy was delivered to both the primary tumor and the peritumoral area at a dose of $40 \mathrm{~Gy}$ in 20 fractions within four weeks. The time interval between neoadjuvant CRT and surgery was two to three weeks.

Clinical response and histopathologic tumor regression after $C R T$. The clinical response after preoperative CRT was evaluated by barium esophagography, endoscopy and computed tomography, and was then graded as a complete response (CR), a partial response (PR), no change (NC) or progressive disease (PD). The pathological response of CRT was evaluated using Mandard tumor regression grade (TRG) (21). Mandard TRG was classified into five categories: TRG1, complete response with absence of residual cancer and fibrosis extending through the wall; TRG2, presence of residual tumor cells scattered through the fibrosis; TRG3, increase in the number of residual cancer cells, with fibrosis predominant; TRG4, residual cancer outgrowing fibrosis; TRG5, absence of regressive changes. We categorized responders as patients with TRG 1 and 2, while non-responders were TRG 3 to 5 .

Lymph node density, peritumoral lymphatic vessel density $(P T L V D)$ and modified Glasgow prognostic score $(m G P S)$. To find useful prognostic markers, we analyzed the association of lymph node density, peritumoral lymphatic vessel density (PTLVD) and modified Glasgow prognostic score (mGPS) with prognosis. Lymph node density is defined as the percentage of positive lymph nodes/total number of excised lymph nodes accordibg to a previous study (22). As podoplanin is a specific marker for lymphatic endothelium, we counted peritumoral lymphatic vessels in four microscopic fields per slide at a magnification of $\mathrm{x} 100$, and termed the average number as peritumoral lymphatic vessel density. mGPS is defined as follows: patients with both an elevated C-reactive protein $(>1.0 \mathrm{mg} / \mathrm{dl})$ and low albumin $(<3.5 \mathrm{~g} / \mathrm{dl})$ were allocated a score of 2 ; patients in whom only C-reactive protein was elevated $(>1.0 \mathrm{mg} / \mathrm{dl})$ were allocated a score of 1 and those with a normal $\mathrm{C}$-reactive protein were allocated a score of 0 (23).

Immunohistochemical analysis of podoplanin (D2-40) and SOX2. FFPE specimens were sliced into 2- to 3- $\mu \mathrm{m}$ sections.
We stained at least three different sections per case to confirm reproducibility. After deparaffinization and dehydration, specimens were brought to a boil in $10 \mathrm{mM}$ sodium citrate buffer for antigen unmasking. Specimens were then blocked and incubated with primary antibody overnight at $4^{\circ} \mathrm{C}$. The antibody was detected using Envision reagents (Envision kit/ HRP, Dako Cytomation, Denmark). Primary antibody D2-40 (mouse monoclonal antibody, clone: D2-40, code no. M3619, Dako Cytomation) for podoplanin and SOX2 primary antibody (rabbit polyclonal antibody, H-65, sc-20088, Santa-Cruz Biotechnology, CA, USA) were used at a dilution of 1:100 for implementation of the labeled streptavidin-biotin method (LASB2 kit/HRP, Dako Cytomation), and 3, 3'-diaminobenzidine (Dako Cytomation). All sections were counterstained with hematoxylin, dehydrated and mounted. Negative controls were run simultaneously. Podoplanin and SOX2 expression was evaluated semi-quantitatively in a blinded fashion without knowledge of clinical and pathological information by a single investigator.

Statistical analysis. Statistical analysis was done using Stat View 5.0 (SAS Institute Inc., Cary, NC). Significant differences were analyzed using the Chi-square test. Overall survival probabilities were calculated from the date of surgery to the date of death, using the Kaplan-Meier product limit method; intergroup differences were determined using the log-rank test. The influence of distant recurrence and survival predictors identified by univariate analysis was assessed by multivariate analysis using Cox's proportional hazards model. P-values $<0.05$ were considered statistically significant.

\section{Results}

Expression of podoplanin and SOX2 after neoadjuvant CRT. Fig. 1 shows immunohistochemical finding of podoplanin and SOX2. Podoplanin expression was mainly observed at the outer edge of residual cancer nests, and at the membranes of these cells. Moreover, stromal cells surrounding residual cancer nests were strongly stained by podoplanin compared to adjacent normal stromal tissue ( $a$ and $b$ ). On the other hand, SOX2 expression was observed in the nucleus and cytoplasm of cancer cells. SOX2 expression seemed to be cancer-specific (c and d). Both podoplanin and SOX2 expression were detected in the basal layer of the adjacent normal mucosa in some cases (Fig. 2). We defined the podoplanin high expression group as cases in which $>50 \%$ of residual cancer cells possessed high stromal immunoreactivity for podoplanin and the high SOX2 expression group as cases with $>50 \%$ of cancer cells. The remaining constituted the low expression groups. Twelve and eight cases were in the high podoplanin and SOX2 group, respectively. There was a significant correlation between podoplanin and SOX2 expression (Chi-square test; $\mathrm{P}=0.0404$ ).

Correlation of podoplanin and SOX2 expression with clinicopathological variables. Table I shows patient characteristics and correlation of the expression of podoplanin and SOX2 with clinicopathological variables. Twenty patients were included in this study. The median age was 68 years (range, 52-77 years) and the male to female ratio was 9:1. The median follow-up period was 20 months (range, 4-109 months). The post-CRT 

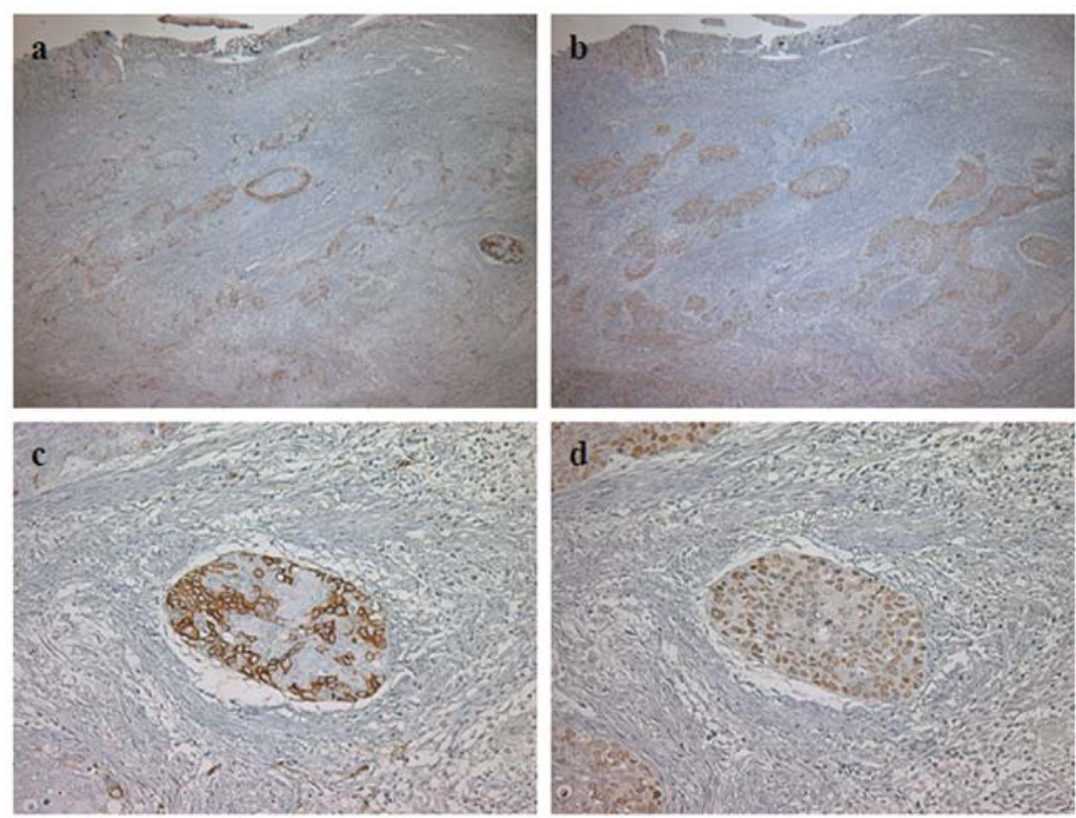

Figure 1. Immunohistochemistry of podoplanin and SOX2. Podoplanin expression was observed at the outer edge of residual cancer nests (a and c). SOX2 expression was observed in the nucleus and cytoplasm of residual cancer cells (b and d). Original magnification: x40 (a and b), 200 (c and d).
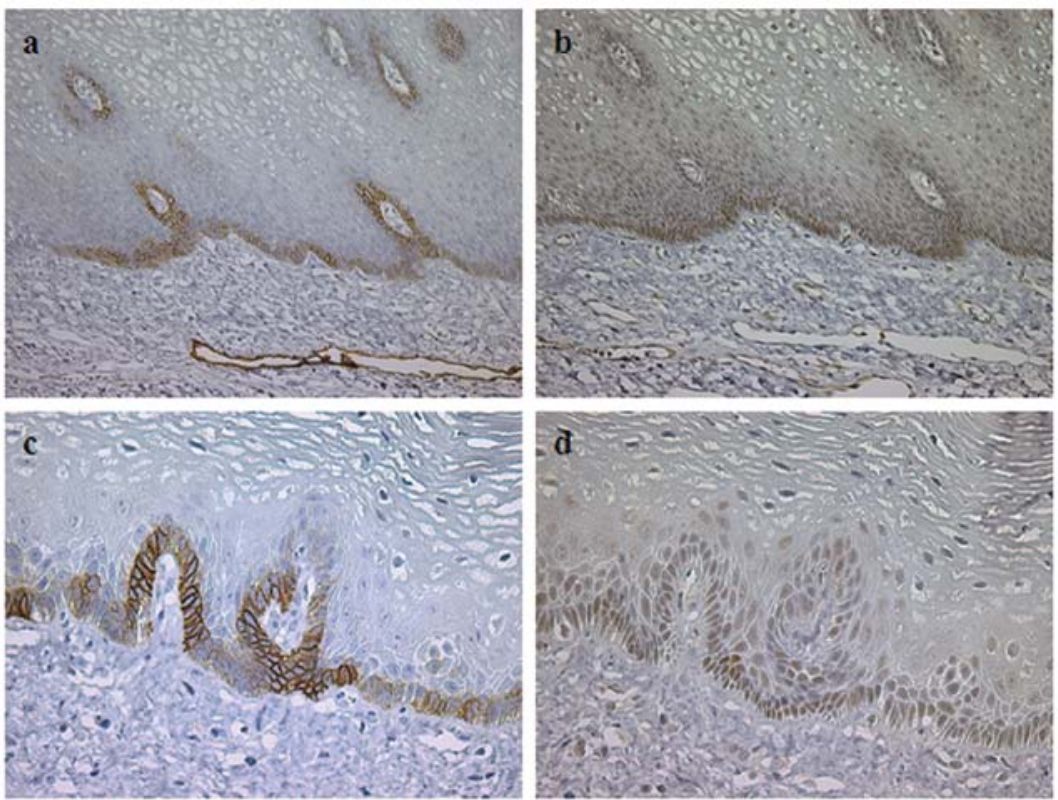

Figure 2. Podoplanin and SOX2 expression in the basal layer of the esophagus. Both podoplanin and SOX2 expression were detected in the basal layer of adjacent normal mucosa. Original magnification: x200 (a and b), x400 (c and d).

pathological T stages were as follows: pT1 $(n=1)$, pT2 $(n=9)$, pT3 $(n=9)$ and pT4 $(n=1)$. Eleven patients $(55 \%)$ had lymph node metastases. Seventeen tumors $(85 \%)$ showed well or moderately differentiated squamous cell carcinoma histology. $\mathrm{R} 0$ resection was done in $80 \%$ of the cases. Clinical response was: NC, 9 patients; PR, 10 patients; CR, 1 patient. TRG was: TRG1, 0 patients; TRG2, 9 patients; TRG3, 7 patients; TRG4, 4 patients; and, TRG5, none. Therefore, 9 patients had a response. High expression of podoplanin was significantly correlated with lymph node metastasis $(\mathrm{P}=0.0277),>20 \%$ of lymph node density, advanced postoperative stage $(\mathrm{P}=0.0062)$ and vascular invasion $(\mathrm{P}=0.0404)$. High expression of SOX2 was significantly correlated with lymphatic $(\mathrm{P}=0.0022)$ and vascular invasion $(\mathrm{P}=0.0004)$, poorly differentiated tumor $(\mathrm{P}=0.0214)$ and incomplete resection $(\mathrm{P}=0.0062)$.

Lymph node density, PTLVD and $m$ GPS. The median value of total dissected lymph node was 13.5 (range, 2-39) and the median value of lymph node density was $5.45 \%$ (range, $0-55.6$ ). We categorized the cases with $>20 \%$ of lymph node density were defined as high lymph node density group, and the remainder as the low group (22). The median value of 
Table I. Patient characteristics and correlation of the expression of podoplanin and SOX2 with clinicopathological variables.

\begin{tabular}{|c|c|c|c|c|c|c|c|}
\hline Variables & Number (\%) & $\begin{array}{l}\text { PDPN low } \\
\quad(\mathrm{n}=8)\end{array}$ & $\begin{array}{l}\text { PDPN high } \\
\quad(\mathrm{n}=12)\end{array}$ & P-value & $\begin{array}{c}\text { SOX2 low } \\
(\mathrm{n}=12)\end{array}$ & $\begin{array}{l}\text { SOX2 high } \\
\quad(\mathrm{n}=8)\end{array}$ & P-value \\
\hline \multicolumn{8}{|l|}{ Gender } \\
\hline Male & $18(90)$ & 7 & 11 & \multirow[t]{2}{*}{0.7609} & 10 & 8 & \multirow[t]{2}{*}{0.2235} \\
\hline Female & $2(10)$ & 1 & 1 & & 2 & 0 & \\
\hline \multicolumn{8}{|l|}{ Age(median: 69 years) } \\
\hline$<69$ & $10(50)$ & 5 & 5 & \multirow[t]{2}{*}{0.3613} & 6 & 4 & \multirow[t]{2}{*}{$>0.9999$} \\
\hline$\geq 69$ & $10(50)$ & 3 & 7 & & 6 & 4 & \\
\hline \multicolumn{8}{|l|}{ T classification } \\
\hline $\mathrm{T} 1 / 2$ & $10(50)$ & 5 & 5 & \multirow[t]{2}{*}{0.3613} & 8 & 2 & \multirow[t]{2}{*}{0.0679} \\
\hline $\mathrm{T} 3 / 4$ & $10(50)$ & 3 & 7 & & 4 & 6 & \\
\hline \multicolumn{8}{|l|}{$\mathrm{N}$ classification } \\
\hline Absent & $9(45)$ & 6 & 3 & \multirow[t]{2}{*}{0.0277} & 7 & 2 & \multirow[t]{2}{*}{0.1421} \\
\hline Present & $11(55)$ & 2 & 9 & & 5 & 6 & \\
\hline \multicolumn{8}{|l|}{ Lymph node density } \\
\hline Low $(<20 \%)$ & $13(65)$ & 8 & 5 & \multirow[t]{2}{*}{0.0074} & 9 & 4 & \multirow[t]{2}{*}{0.2508} \\
\hline High $(\geq 20 \%)$ & $7(35)$ & 0 & 7 & & 3 & 4 & \\
\hline \multicolumn{8}{|l|}{ Postoperative stage } \\
\hline $\mathrm{I} / \mathrm{II}$ & $10(50)$ & 7 & 3 & \multirow[t]{2}{*}{0.0062} & 8 & 2 & \multirow[t]{2}{*}{0.0679} \\
\hline III/IV & $10(50)$ & 1 & 9 & & 4 & 6 & \\
\hline \multicolumn{8}{|l|}{ Lymphatic invasion } \\
\hline ly $0 / 1$ & $13(20)$ & 7 & 6 & \multirow[t]{2}{*}{0.0850} & 11 & 2 & \multirow[t]{2}{*}{0.0022} \\
\hline ly $2 / 3$ & $7(80)$ & 1 & 6 & & 1 & 6 & \\
\hline \multicolumn{8}{|l|}{ Vascular invasion } \\
\hline Absent & $12(60)$ & 7 & 5 & \multirow[t]{2}{*}{0.0404} & 11 & 1 & \multirow[t]{2}{*}{0.0004} \\
\hline Present & $8(40)$ & 1 & 7 & & 1 & 7 & \\
\hline \multicolumn{8}{|l|}{ Histology } \\
\hline Well/moderately & $17(85)$ & 8 & 9 & \multirow{2}{*}{0.1250} & 12 & 5 & \multirow[t]{2}{*}{0.0214} \\
\hline Poorly & $3(15)$ & 0 & 3 & & 0 & 3 & \\
\hline \multicolumn{8}{|l|}{$\mathrm{R} 0$ resection } \\
\hline Yes & $16(80)$ & 8 & 8 & 0.0679 & 12 & 4 & 0.0062 \\
\hline No & $4(20)$ & 0 & 4 & & 0 & 4 & \\
\hline Mandard TRG & & & & & & & \\
\hline Grade 3-5: non responder & $\mathrm{r} \quad 11(55)$ & 3 & 8 & 0.1990 & 5 & 6 & 0.1356 \\
\hline Grade 1, 2: responder & $9(45)$ & 5 & 4 & & 7 & 2 & \\
\hline
\end{tabular}

$\mathrm{T}$ and $\mathrm{N}$ categories indicate extent of the tumor and presence of lymph node metastasis. R0 resection, complete resection with no microscopic residual cancer. Lymph node density, the percentage of positive lymph nodes/total number of excised lymph nodes. PDPN, podoplanin.

PTLVD was 16.33 (range, 5-37). We categorized the cases with more than the median value as the high PTLVD group, and the remaining as the low group. mGPS was: mGPS 2,5 patients; mGPS 1,6 patients and mGPS 0,9 patients.

Predictive value of podoplanin and SOX2 expression for overall survival. Fig. 3 shows overall survival according to podoplanin, or SOX2 expression using the Kaplan-Meier product limit method. Cases with high expression of podoplanin showed significantly poorer overall survival than cases with low expression $(\mathrm{P}=0.0125)$. Cases with both low expression of podoplanin and SOX2 had significantly better prognosis $(\mathrm{P}=0.0187)$.
Cox's univariate proportional hazard analysis showed that high lymph node density, $>16$ of PTLVD and high podoplanin expression were significantly associated with poor overall survival $(\mathrm{P}=0.0303, \mathrm{P}=0.0498$ and $\mathrm{P}=0.0199$, respectively). In Cox's multivariate hazard model analysis, only high PTLVD independently predicted poor survival $(\mathrm{P}=0.0333$ ) (Table II).

\section{Discussion}

This preliminary study is the first to report an association of podoplanin and SOX2 expression with clinical outcome in ESCC after neoadjuvant CRT. Rahadiani et al reported that 
Table II. Uni- and multivariate analyses for predictors of survival.

\begin{tabular}{|c|c|c|c|}
\hline Variables & Hazard ratio & $95 \% \mathrm{CI}$ & P-value \\
\hline \multicolumn{4}{|l|}{ Univariate analysis } \\
\hline Age $(<69$ vs. $\geq 69)$ & 0.331 & $0.237-2.192$ & 0.564 \\
\hline $\mathrm{T}$ classification (T1/2 vs. T3/4) & 0.340 & $0.453-4.314$ & 0.560 \\
\hline $\mathrm{N}$ classification (absent vs. present) & 1.947 & $0.135-1.400$ & 0.163 \\
\hline Lymph node density (low vs. high) & 4.691 & $1.138-13.269$ & 0.030 \\
\hline Lymphatic invasion (ly 0/1 vs. $2 / 3$ ) & 0.986 & $0.181-1.750$ & 0.321 \\
\hline Vascular invasion (absent vs. present) & 0.506 & $0.214-2.054$ & 0.477 \\
\hline Postoperative stage (I/II vs. III/IV) & 1.587 & $0.155-1.500$ & 0.208 \\
\hline R0 (yes vs. no) & 1.091 & $0.134-1.843$ & 0.296 \\
\hline Mandard TRG (non-responder vs. responder) & 2.121 & $0.746-7.280$ & 0.145 \\
\hline Preoperative SCC (<1.5 vs. $\geq 1.5 \mathrm{ng} / \mathrm{ml})$ & 0.292 & $0.424-4.540$ & 0.589 \\
\hline Modified GPS (0/1 vs. 2) & 1.219 & $0.156-1.681$ & 0.270 \\
\hline PTLVD ( $\leq 16$ vs. $>16)$ & 3.848 & $1.001-11.625$ & 0.049 \\
\hline Podoplanin (low vs. high) & 5.417 & $1.259-14.752$ & 0.019 \\
\hline SOX2 (low vs. high) & 1.784 & $0.704-6.365$ & 0.181 \\
\hline \multicolumn{4}{|l|}{ Multivariate analysis } \\
\hline Lymph node density (low vs. high) & 0.129 & $0.285-6.174$ & 0.719 \\
\hline PTLVD ( $\leq 16$ vs. $>16)$ & 4.529 & $1.117-14.629$ & 0.033 \\
\hline Podoplanin (low vs. high) & 3.131 & $0.856-20.842$ & 0.076 \\
\hline
\end{tabular}

TRG, tumor regression grade; PTLVD, peritumoral lympatic vessel density; GPS, Glasgow prognostic score; CI, confidence interval.
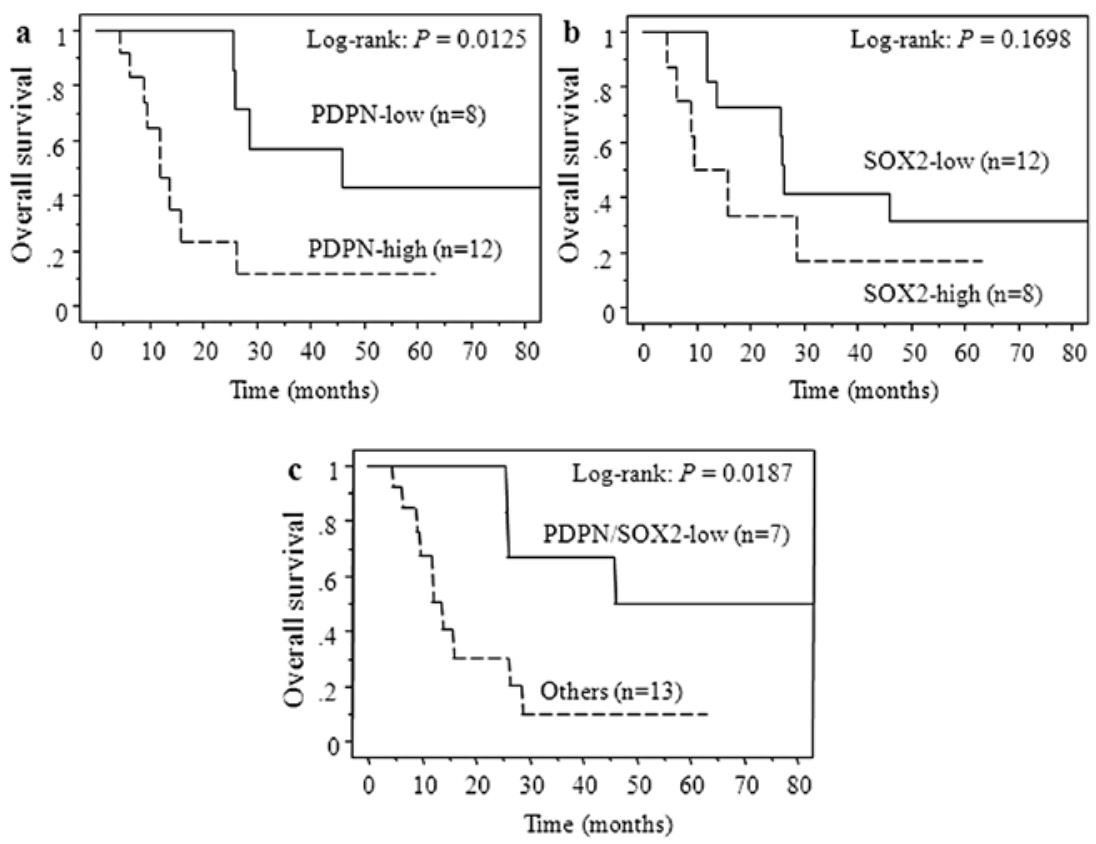

Figure 3. Overall survival according to low and high expression of podoplanin and SOX2. The Kaplan-Meier method was used to generate overall survival curves. Cases with high expression of podoplanin (a) but not SOX2 (b) had significantly poorer overall survival compared to those with low expression. Cases with both low expressions of podoplanin and SOX2 had significantly better prognosis (c).

podoplanin-positive cells showed high levels of tumorigenic activity, and that knockdown of podoplanin expression reduced resistance to anticancer drugs, and decreased invasiveness and tumorigenic activity (9). Shimada et al reported that podoplanin expression reflected the immature status in lung squamous cell carcinoma cells. They also found that immunostaining of podoplanin, CD44 and p63 and analysis of the hierarchical distribution patterns of the markers in the tumor nests revealed 
cells with a capacity for further maturation (24). Long et al demonstrated that SOX2 was implicated in the maintenance of pluripotency and esophageal epithelial development, and that it was preferentially expressed in ESCC (13). From these findings, we hypothesized that podoplanin and SOX2 expression were involved in tumor growth and invasion in ESCC after neoadjuvant CRT. We observed both podoplanin and SOX2 expression in the basal layer of adjacent normal mucosa after CRT. This finding suggests both podoplanin and SOX2 may play roles in the development of esophageal epithelium. Moreover, there was a significant correlation between podoplanin and SOX2 expression in immunohistochemical analysis $(\mathrm{P}=0.0404)$. Podoplanin expression was observed in the membranes of residual cancer cells at the outer edge of residual cancer nests, but not in the central region. Our finding was similar to previous reports of squamous cell carcinoma in head and neck, and the oral cavity. In addition, we found that residual cancer with high podoplanin expression showed a high immunoreactivity in stromal tissue. These results suggest that cancer cells and stroma might interact through podoplanin, and that podoplanin expression in stromal tissue surrounding cancer cells might be increased by CRT. Wicki et al reported that podoplanin expression in breast cancer cells was induced by epidermal growth factor, basic fibroblast growth factor and tumor necrosis factor $\alpha$ in in vitro study (14). On the other hand, SOX2 expression was detected in residual cancer cells after CRT, and was rarely detected in stromal cells. The specificity for residual ESCC may be diagnostically useful for both the accurate evaluation of a patient's response after CRT and the detection of micrometastases in lymph nodes. We observed that high expression of podoplanin was significantly correlated with lymph node metastasis, vascular invasion and poor prognosis, while, high expression of SOX2 was lymphatic, vascular invasion, poorly differentiated tumor and pathological incomplete resection. However, correlation of these expression levels with TRG multivariate analysis identified high PTLVD as independent risk factors for predicting poor prognosis. Several authors have reported that high peri- or intra-tumoral lymphatic vessel density is correlated with lymph node metastasis and poor prognosis in oral, head and neck squamous cell carcinoma and breast cancer (25-27). Our result was similar to previous studies, and suggested that PTLVD might be also associated with poor prognosis in patients with ESCC with neoadjuvant CRT. In conclusion, our results suggest that podoplanin and SOX2 expression levels may be useful prognostic markers for patients with ESCC after neoadjuvant CRT. However, data in this study should be interpreted with some caution. The major limitation is the small number of patients, and the retrospective nature of the study. We think that a larger study population and a long-term follow-up will allow us to validate our conclusions.

\section{References}

1. Vizcaino AP, Moreno V, Lambert R, et al: Time trends incidence of both major histologic types of esophageal carcinomas in selected countries, 1973-1995. Int J Cancer 99: 860-868, 2002.

2. Lv J, Cao XF, Zhu B, et al: Effect of neoadjuvant chemoradiotherapy on prognosis and surgery for esophageal carcinoma. World J Gastroenterol 15: 4962-4968, 2009.

3. Stahl M, Stuschke M, Lehmann N, et al: Chemoradiation with and without surgery in patients with locally advanced squamous cell carcinoma of the esophagus. J Clin Oncol 23: 2310-2317, 2005.
4. Bedenne L, Michel P, Bouche O, et al: Chemoradiation followed by surgery compared with chemoradiation alone in squamous cancer of the esophagus: FFCD 9102. J Clin Oncol 25: 1160-1168, 2007.

5. Gebski V, Burmeister B, Smithers BM, et al: Survival benefits from neoadjuvant chemoradiotherapy or chemotherapy in oesophageal carcinoma: a meta-analysis. Lancet Oncol 8: 226-234, 2007.

6. Reya T, Morrison SJ, Clarke MF, et al: Stem cells, cancer, and cancer stem cells. Nature 414: 105-111, 2001.

7. Vermeulen L, Sprick MR, Kemper K, et al: Cancer stem cells-old concepts, new insights. Cell Death Differ 15: 947-958, 2008.

8. Atsumi N, Ishii G, Kojima M, et al: Podoplanin, a novel marker of tumor-initiating cells in human squamous cell carcinoma A431. Biochem Biophys Res Commun 373: 36-41, 2008.

9. Rahadiani N, Ikeda J, Makino T, et al: Tumorigenic role of podoplanin in esophageal squamous-cell carcinoma. Ann Surg Oncol 17: 1311-1323, 2010

10. Raica M, Cimpean AM and Ribatti D: The role of podoplanin in tumor progression and metastasis. Anticancer Res 28: 2997-3006, 2008.

11. Kreppel M, Scheer M, Drebber U, et al: Impact of podoplanin expression in oral squamous cell carcinoma: clinical and histopathologic correlations. Virchows Arch 456: 473-482, 2010.

12. Rodrigo JP, Garcia-Carracedo D, Gonzalez MV, et al: Podoplanin expression in the development and progression of laryngeal squamous cell carcinomas. Mol Cancer 9: 48, 2010.

13. Long KB and Hornick JL: SOX2 is highly expressed in squamous cell carcinomas of the gastrointestinal tract. Hum Pathol 40: 1768-1773, 2009.

14. Wicki A, Lehembre F, Wick N, et al: Tumor invasion in the absence of epithelial-mesenchymal transition: podoplaninmediated remodeling of the actin cytoskeleton. Cancer Cell 9: 261-272, 2006.

15. Cueni LN, Hegyi I, Shin JW, et al: Tumor lymphangiogenesis and metastasis to lymph nodes induced by cancer cell expression of podoplanin. Am J Pathol 177: 1004-1016, 2010.

16. Schacht V, Ramirez MI, Hong YK, et al: T1alpha/podoplanin deficiency disrupts normal lymphatic vasculature formation and causes lymphedema. EMBO J 22: 3546-3556, 2003.

17. Sleeman JP and Thiele W: Tumor metastasis and the lymphatic vasculature. Int J Cancer 125: 2747-2756, 2009.

18. Yamanaka S: Strategies and new developments in the generation of patient-specific pluripotent stem cells. Cell Stem Cell 1: 39-49, 2007.

19. Bass AJ, Watanabe $\mathrm{H}$, Mermel $\mathrm{CH}$, et al: SOX2 is an amplified lineage-survival oncogene in lung and esophageal squamous cell carcinomas. Nat Genet 41: 1238-1242, 2009.

20. Que J, Okubo T, Goldenring JR, et al: Multiple dose-dependent roles for Sox 2 in the patterning and differentiation of anterior foregut endoderm. Development 134: 2521-2531, 2007.

21. Mandard AM, Dalibard F, Mandard JC, et al: Pathologic assessment of tumor regression after preoperative chemoradiotherapy of esophageal carcinoma. Clinicopathologic correlations. Cancer 73: 2680-2686, 1994.

22. Kassouf W, Agarwal PK, Herr HW, et al: Lymph node density is superior to TNM nodal status in predicting disease-specific survival after radical cystectomy for bladder cancer: analysis of pooled data from MDACC and MSKCC. J Clin Oncol 26: 121-126, 2008.

23. McMillan DC, Crozier JE, Canna K, et al: Evaluation of an inflammation-based prognostic score (GPS) in patients undergoing resection for colon and rectal cancer. Int J Colorectal Dis 22: 881-886, 2007.

24. Shimada Y, Ishii G, Nagai K, et al: Expression of podoplanin, CD44, and p63 in squamous cell carcinoma of the lung. Cancer Sci 100: 2054-2059, 2009.

25. Kyzas PA, Geleff S, Batistatou A, et al: Evidence for lymphangiogenesis and its prognostic implications in head and neck squamous cell carcinoma. J Pathol 206: 170-177, 2005.

26. Longatto Filho A, Oliveira TG, Pinheiro C, et al: How useful is the assessment of lymphatic vascular density in oral carcinoma prognosis? World J Surg Oncol 5: 140, 2007.

27. Debald M, Polcher M, Flucke U, et al: Increased detection of lymphatic vessel invasion by D2-40 (podoplanin) in early breast cancer: possible influence on patient selection for accelerated partial breast irradiation. Int J Radiat Oncol Biol Phys 77: 1128-1133, 2010. 